\title{
Review
}

\section{Periorbital hyperpigmentation, a dermatologic condition having a strong geographic and ethnic determinism}

\author{
Felicia Andrei ${ }^{1}$, Daciana Grujic ${ }^{2 *}$, Cristina Lazar ${ }^{3}$ and Anca Dragomirescu ${ }^{1}$ \\ 1 Department of Dermatopharmacy and Cosmetology, Faculty of Pharmacy, University of Medicine and \\ Pharmacy "Victor Babes", Timisoara, Romania; felicia.andrei@umft.ro \\ 2 Department of Plastic Surgery, Faculty of Medicine, University of Medicine and Pharmacy "Victor Babes", \\ Timisoara, Romania; grujic.daciana@umft.ro \\ 3 Master Degree in the Department of Dermatopharmacy and Cosmetology, Faculty of Pharmacy, University \\ of Medicine and Pharmacy "Victor Babes", Timisoara, Romania \\ * Correspondence: grujic.daciana@umft.ro; Tel.: +40-726-503-896
}

\begin{abstract}
POH}$ (Peri Orbital Hyperpigmentation) represents a minor clinical entity that attracts immense aesthetic damages and it generates social integration difficulties. This review focuses on the etiopathogenic causes of this entity, differentiating and reclassifying this defect as having, on the one hand, genetic causes of melanic hyperproduction - for Fitzpatrick cutaneous phototypes IV and V - and, on the other hand, both genetic and acquired vascular causes, in individuals with light-coloured skin phototypes. Hence, there is a big difference in the field of pathogenic treatment, for the two entities. In addition, this study notes the direct relationship between skin aging and $\mathrm{POH}$, especially for aquired vascular causes. In this reasoning, other aesthetic deficiencies of the skin in the palpebral area should be also considered, like: blepharochalasis, wrinkles, the anatomical causes of the lower eyelid shading, symmetrical or asymmetric suborbital oedema. All of these issues will complicate the therapeutic decision and subsidiary, the pharmaceutical advice. In this context, the review shows the guidelines for a honest councelling of the patients, pointing the efficiency limit for the topical pharmaceutical medication (depigmentants, exfoliants) versus the necessity of minimally invasive or/ and surgical treatments (in blefarochalasis).
\end{abstract}

Keywords: hyperpigmentation; palpebral region; geographic skin differences; ethnic predisposition; skin disorder;

\section{Introduction}

The eyes and their gaze always aroused interest both for aesthetic and verbal human communication. The appearance and their expression are in direct relationship with the health, but also the beauty of a person. Standards of beauty, extremely changeable during the human history in terms of body contours, have remained constant for the luminosity and the eye expressiveness. There are many issues that may occur at this level but only one of them will be exhaustively presented in this review.

Named most often Periorbital hyperpigmentation ( $\mathrm{POH})$, the deficiency seems to have in literature many names such as: Idiopathic Cutaneous Hypercromia in the Orbital Region (ICHOR) [1], Infraorbital dark circles [2], Cutaneous Idiopathic Hypercromia of the Orbital Region (CIHOR) [3], or Periorbital Melanosis [4]. Lack of unique names would not be a serious problem in itself, but behind these inconsistencies are usually different etiopatogenic approaches, thus implicitly pathogenic targeted treatment. In a simple documentary search on the internet there is a wealth of multidisciplinary approaches to the problem, some contradictory, so that this pathology has been rolled away from ophthalmologists and dermatologists to plastic surgery and laser therapy, back to 
pharmaceutical research. The lack of a uniform approach comes from the fact that there is no large addressability to the practitioner for this situation, many patients continue considering it as a fatality, an aesthetic defect that cannot be solved therapeutically. This hazy about the best treatment, gave wings to the cosmetics and camouflage products to propose "miracle" solutions. If to all this adds the possible confusions with other pathologies, involving defective aesthetic of the eyelid region: the characteristic age caused blefarochalosis and the eyelid edema (resulting from fluid retention, sometimes near normal in the premenstrual syndrome PMS), we have the full picture of aesthetic defects of the eyelid, able to bring a grater confusion in the problem.

Framing correct the disease in primary (genetic) and secondary, further complicates the understanding of this pathology, given to the fact that there are late penetrating genetic defects. However, familial aggregation of this situation clearly tilted the balance towards a genetic determinism [1]. Secondary causes can be more easily identified, in that it may present with asymmetry (different intensity for the two lower eyelids), or be vascular, post inflammatory, hormonal, caused by prolonged exposure to sun, aging or even a apparently darker (shadow) by fat pseudo hernia of the eye [1].

The prevalence of $\mathrm{POH}$ is also a relative value, because most studies observed a great variability of clinical form, color, appearance and incidence depending on the skin phototype. By $\mathrm{R}$. Sarkar, citing other recent studies, the population of Singapore most common form is vascular (41\%), rather staining erythematous, sometimes with telangiectasias, or highlighted blood vessels, favored by the specific epidermal aging atrophy. In this classification, the hierarchy continue with the constitutional due influence (38\%), characterized by melanic hyperpigmentation, and only $12 \%$ of patients enrolled with $\mathrm{POH}$ can assert a postinflamatory causal hyperpigmentation ${ }^{1}$. The shown classification is just one facet of the problem, seen from a single prism. Therefore we can talk about a large geographical variability of the phenomenon. According to other studies, the Chinese population is rather touched by the vascular form of the $\mathrm{POH}$, while the Indian populations, by the melanic cause [5]. More than that, for the Indian population the stains of $\mathrm{POH}$ are bounded by an anatomically suborbital net line (fold) [4], and for the Japanese population, the hystopathological analysis noted a dermal melanocitosis [6].

The very striking feature of the addiction to the skin phototype is observed by other studies [7, 8].The Indian authors incriminates for over half of the cases, the constitutional melanic cause (51\%) [9], and in addition the Korean researchers note the anatomical cause anatomical, the Orbicularis oculii muscle laxity, able to create a shadow, a dark aspect of the subocular region [10].

\section{Pathogenesis}

On the one hand, the literature of European and part of the North American authors include periorbital hyperchromia in the process of chronologic aging, with implications resulting from blood and vascular defects, as an acquired process, and not necessarily due to an abnormality of genetic cause, primary. This point of view is not caused by a limited vision of the problem, but that subjects in the geographical area are predominantly light skin phototype (I, II and III, mostly). Suppa M. highlights the obvious relationship between the eyelid pigmentation and extrinsec factors composing the aging processes: UV exposure, smoking, vascular problems [10]. Mary L Lupo et al. considers the important role of intrinsec aging in the pathogenesis of periorbital eyes pigmentation, proposing for this reason a new topical treatment, based on human growth factors, cytokines, caffeine and Glycyrrhetinic acid [11]. Their vision practically admits both the melanin factor in the pathogenesis - targeted by the Glycyrrhetinic acid (an inhibitor of the melanogenesis) and also the vascular factors, therefore including caffeine in a pharmaceutical product.

Not far from their point of view is that of A. Wagner et al., attributing to the pathology exclusively aesthetic eyelid causes related to aging [12] and to these considerations are added those of Sydnay R. et al. which bring the point of view of a plastic operating surgeon, anchored in the study of human anatomy. They see the dark suborbital appearance as an expression of the soft tissue retraction, an age-related process, leading to the formation of subocular depressions and the creation of a shadow image. In this process contributes both the thinning of the epidermis and loss of the 
dermal fermity through water and hyaluronic acid depreciation, bone resorption, sadder elasticity by imminent losses of collagen and elastin in the dermis, herniation of suboculare fat and not least, decreased muscle tone around the eyes, in a word: skin laxity. However, they admit also an excessive subocular melanin synthesis, as an expression of senile pigmentation [13]. All these effects have a negative impact on the aged patient's psyche [14].

On the other hand, the literature of Asian and Sudamerican authors joins the mainstream and put as a primary cause in the pathogenesis of the periorbital hypercoloration rather the melanic, constitutional mechanism, called melanosis. Here it is not a single-minded vision, but the underlining of a specific skin fototipe. Kanika $S$ et al. add the role of post inflammatory pigmentations in the aggravation and intensification of this pathology [15], while Momosawa et al. assigned exclusively to the melanic cause this process, proposing a pretreatment with a mix of tretinoin and hydroquinone, previous to a laser procedure [16].

Michèle Verschoore et al., in a study conducted on patients, assessing hemoglobin and melanin mexametry in the subocular region in patients with $\mathrm{POH}$, fore melanic cause not excluding endogenous processes, like specific aging factors [17].

Corroborating the two current informations, regardless the skin phototype, we can conclude which are the possible causes of $\mathrm{POH}$ :

- melanic constitutional (genetic) pigmentation,

- post-inflammatory pigmentation (chronic sinusitis),

- an atrophy of the periorbital skin, caused by aging, leading to a skin transparency

- venous congestion,

- accumulation of hemoglobin and oxygenated hemoglobin,

- storage of haemosiderin resulting by erythrocytes ruptures in pacients with capillar fragility,

- anatomical causes: ptosis of the orbital fat, specific, age caused, subocular soft tissue retraction.

Compared to these favorable causes of appearance, there are many other situations that precipitate and exacerbate this phenomenon (Table 1)

Table 1. Precipitating and aggravating factors of periorbital hyperpigmentation and their mechanism of action

\begin{tabular}{ll}
\hline Aggravating factor & The developped mechanism \\
\hline Excessive exposure to UV & Increased melanin synthesis \\
Abuse of alcohol and tobacco & Accelerates the extrinsic aging process and hemolysis \\
Pregnancy and contraceptive medication & Stimulates the melanin synthesis \\
Stress and insufficient rest & Induces erythrocyte hemolysis \\
\hline
\end{tabular}

Therefore, the literature describes the one hand a certain type of $\mathrm{POH}$, rather vascular, common in people with phototype I, II and III, having erythematous shades, on the other hand another type more common in persons with skin phototype IV and V (with dark skin), melanic caused. This latter type of dark circles shows shades of brown. It is important to know the prevailing pathogenesis to initiate the most appropriate cosmeto-dermatologic treatment. Concrete Mexameter ${ }^{\circledR}$ measurements can distinguish the two types of $\mathrm{POH}$.

\section{The relationship between skin aging and $\mathrm{POH}$}

This As we stated previously, skin aging stresses and advances the signs of $\mathrm{POH}$, but looking more closely to this issue, we find that there is a double meaning, because the installation of the periorbital hyperpigmentation by any pathogenic mechanism is able to close the vicious circle of this aesthetic defect by lowering the self-esteem, resignation and giving up any possible dermatological cosmetic care [14].

Almost all of the pathophysiological links of aging generally have a response on the aesthetic eyelid pathology. We have systematized this process in the form of Table 2 
Table 2. The systemic aging and its consequences on the eyelid structure

\section{General (systemic) fenomena of aging Cellular senescence with telomeric loss, escalated by stress, UV exposure and chronological aging with every cell division $[18,19]$. \\ Decreasing number of stem cells and cells with potential division}

The production of toxic free radicals

Protein and collagen glycation [22].

Biological clock's altering

Decreased estrogen production, menopause [25].

Decreased androgenic production [25].

The increase in cortisol secretion, stress, insomnia $[27,28]$.

Sarcopenia touching elderly people by suppression of the androgen stimulation in both sexes

Cumulative effect of sun exposures, age dependent, the face being permanently exposed
The consequence on the eyelid and the skin

Senescent keratinocytes and fibroblasts diminished their activity and have a reduced viability. Delayed healing after the intervention of blepharoplasty.

Atypical epidermal atrophy and exaggerated skin apoptosis [20]. The decrease of the epidermal mitosis in the basal layer. Highly visible epidermal transparency on the suborbital level. Highlighting of subocular deep pygmentations

Advancing of previously described aging processes and accelerating telomeric destructions [21]. Collagen hardening, loss of elasticity of skin on eyelid level [23, 24]. Blefarochalasis.

The decrease in the rate of cellular rhythm and synthesis (collagen, elastine, hyaluronic acid, ceramide, immunoglobulin, cytokines, growth factors). Atrophy of the epidermis, eyelid transparency in the dermis, loss of elasticity, eyelid wrinkles [23-25].

Slowing down the rate of division of the keratinocytes. Transparency and atrophy of eyelid. Osteoporosis, (zygomatic) bone retraction, stuffy aspect of orbits $[13,26]$.

Sebaceous secretion depression, which leads to skin dehydration. Decreased elasticity of eyelid, loss of skin turgor $[25,26]$.

Decrease the hours of sleep and thus increase wakefulness. Inhibited collagen production (protein synthesis, in general). Cortisol is a lytic, catabolic hormone for the proteins [27].

Weakening of the facial muscle, producing the gravitational wrinkles, decreasing the muscle tone of orbicularis oculii. Skin retraction of the lower eyelids. Subocular shadow [13].

Hyperpigmentations, increased subocular melanic agregations [29].

Pathophysiologic processes above are valid for all skin prototypes; only people with dark skin can mask under the subocular melanosis the presence of other colorations. Moreover, the literature writes about different patterns of skin aging for various human races. It is irrefutable that the slower aging is described to be for Asian race people, followed by the african-american population [30].

\section{Periorbital hyperpigmentation and suborbital edema}

Often, there may be a combination of the two entities, but more frequently, confusion, maintained, to some extent, by cosmetics and pharmaceutical or non-pharmaceutical products, presented to the general public as "products for the eyes". 
Common causes of edema of the lower eyelids are represented by fluid retention in the premenstrual syndrome, in which case a possible ptosis of suborbital fat worsen the clinical appearance. The tendency to edema has a constitutional character, but beyond this can stand the predisposition to an increased estrogen-progestogen hormone status.

Internal pathology, the second cause of suborbital edema is due mainly to kidney conditions, and edema resolves in this case by diuretic therapy, without requiring to topical treatment or cosmetic surgery. To have a complete picture of eyelid edema, must be evaluated also the other cases reported in the literature.

Hyperthyroidism is a situation where swelling can be asymmetrical, with associated erythematic upper eyelids. Hystological, it is noteworthy in this case, an expansion of lymphatic vessels at the eyelid, with or without deposits of mucopolysaccharides at that level. Edema is resistant to anti-thyroid medication and is pathognomonic for this dysfunction [31].

Another major cause of the eyelid edema is allergic, but it is to underline that these are situations with relatively rapid development and transient. Nasal mucosa allergies may have an eye echo, causing important edema of the eyelids, and even keratoconjunctivites [32]. Accompanying symptoms are redness, excessive lacrimation, blurred vision, local itching, which distinguishes him from non- allergic eyelid edema, without the immunologic intervention [33]. Angioedema is a known cause of eyelid edema, but the brutality, speed and intensity of the phenomenon eliminates any confusion in the diagnostics [34].

Numerous iatrogenic causes, drugs, including: the treatment with Sirolimus, applied after a kidney transplant as an immunosuppressant for the prevention of organ rejection [35], the pharmacotherapy with Imatinib, applied in gastrointestinal tumor pathology [36] , after a specific tyrosine-kinase inhibitor treatment, Glivec [37], as well as after administration of Olanzapine, where the edema is symmetrical [38].

The anatomical cause of the apparent eyelid edema, produced in reality by the bulging of the capsulo-palpebral fascia, has strictly a surgical treatment, case by case, removing or repositioning the subocular fat [39].

Lastly, it should be remembered the infectious diseases causes of eyelid edema, and remarked that they are transitory, being remised with the complete cure of the disease. One such example was reported for mononucleosis. In this case, points to the lack of pruritus and erythema (which would cause an allergic dermatitis) but evocative presence of fever [40]

\section{Treatment options}

Despite the fact that at present, the $\mathrm{POH}$ causes are known, even we can divide them in three main categories: vascular (more common in light skin phototypes), melanic (rather in dark phototypes) and anatomical, but we cannot confirm the existence of truly effective treatment methods in the studied literature. The first step for a good therapeutic approach would be to give a correct classification for this aesthetic defect, based on a precise case history and noting the context that is grafted in (skin color, allergic ground, added drugs and anatomical conformation). The vascular cause is resistant to a dermocosmetic topic treatment, as states with excessive liberality in the pharmaceutical market, but these patients are ideal candidates to laser therapy [41, 42].

Regarding the suborbital hyperpigmentations of melanic cause there are some active depigmentant substances (tyrosinase inhibitors) proposed for their lightening role, but the depth and intensity of this pigmentation rather requests a chemical peeling [43]. The combination of peeling acids is quoted to have efficacy (3.75\% trichloroacetic acid plus lactic acid $15 \%$ ), but we must underline that they improve the facial appearance for a specified period of 4- 6 months. The anatomical cause is solved strictly surgical by filling/augmentation with fat or with hyaluronic acid $[44,45]$

The analysis of data on the clinical-evolutionary aspects of periorbital hyperpigmentation showed us once again minor differences related to cutaneous skin phototypes which involve major aesthetic damages and social integration problems. In this direction, it can be argued that the Asian 
population has much slower skin-aging capacities compared to the European population. In this last regard, the determinants are whether genetic or linked to dietary habits (Asian soy diet rich in phytoestrogens - the Genistein). [46]

This study attempted to differentiate between vascular and melanic causes for the hyperpigmentation of the palpebral region. This would be important because in this specific case the aesthetic and cosmetic treatment is predominantly based on automedication and publicity. In the cosmetic field the advertising is not always honestly suggested and often not based on scientific truth. [47]

Although the list of natural tyrosinase-blocking depigmenting compounds is always open to researchers, their transposition into industrial practice appears late. [48]

\section{Conclusions}

Although known long ago and recently reclassified by skin prototype and primordial mechanism (at least resulting from geographical and ethnic differences), however effective therapeutic approach is still to be expected. An important and necessary step for the medical-pharmaceutical practice could be, at least, the correct framing of the $\mathrm{POH}$ and to eliminate from the diagnostic reasoning the anatomical causes. The cosmetic medical treatment is merely a time delay and has a depressing influence on the patient's psychic.

With the globalization, this phenomenon can be complicated, and also, clinically and etiologically much more difficult to classify or separate.

Author Contributions: Conceptualization, A.D. and F.A.; Investigation, C.L.; Writing-Original Draft Preparation, D.G.; Writing-Review \& Editing, F.A.; Supervision, A.D.

Funding: "This research received no external funding"

Conflicts of Interest: "The authors declare no conflict of interest."

\section{References}

1. Sarkar, R. Idiopathic Cutaneous Hyperchromia at the Orbital Region or Periorbital Hyperpigmentation. J Cutan Aesthet Surg 2012, 5(3), 183-184

2. Roh, M.R.; Chung, K.Y. Infraorbital dark circles: definition, causes, and treatment options. Dermatol Surg 2009, 35(8), 1163-71, DOI:10.1111/j.1524-4725.2009.01213.x.

3. Graziosi, A.C.; Quaresma, M.R.; Michalany, N.S.; Ferreira, L.M. Cutaneous idiopathic hyperchromia of the orbital region (CIHOR): a histopathological study. Aesthetic Plast Surg 2013, 37(2), 434 - 8, DOI: 10.1007/s00266-012-0048-2.

4. Malakar, S.; Lahiri, K.; Banerjee, U.; Mondal, S.; Sarangi, S. Periorbital melanosis is an extension of pigmentary demarcation line-F on face. Indian J Dermatol Venereol Leprol 2007, 73(5), 323-5

5. Ranu, H.; Thng, S.; Goh, B.K.; Burger, A.; Goh, C.L. Periorbital hyperpigmentation in Asians: an epidemiologic study and a proposed classification. Dermatol Surg 2011, 37(9), 1297-303, DOI: 10.1111/j.1524-4725.2011.02065.x.

6. Watanabe, S.; Nakai, K.; Ohnishi, T. Condition known as "dark rings under the eyes" in the Japanese population is a kind of dermal melanocytosis which can be successfully treated by Q-switched ruby laser. Dermatol Surg 2006, 32(6), 785-9, DOI: 10.1111/j.1524-4725.2006.32161.x.

7. Roberts, W.E. Periorbital hyperpigmentation: review of etiology, medical evaluation, and aesthetic treatment. J Drugs Dermatol. 2014, 13(4), 472-82. 
8. Ho, S.G.; Chan, H.H. The Asian dermatologic patient: review of common pigmentary disorders and cutaneous diseases. Am J Clin Dermatol. 2009, 10(3), 153-68, DOI: 10.2165/00128071-200910030-00002.

9. Sheth, P.B.; Shah, H.A.; Dave, J.N. Periorbital hyperpigmentation: a study of its prevalence, common causative factors and its association with personal habits and other disorders. Indian J Dermatol 2014, 59(2), 151-7, DOI:10.4103/0019-5154.127675.

10. Suppa, M.; Elliott, F.; Mikeljevic, J.S.; Mukasa, Y.; Chan, M.; Leake, S.; Karpavicius, B.; Haynes, S.; Bakker, E.; Peris, K.; Barrett, J.H.; Bishop, D.T.; Newton Bishop, J.A. The determinants of periorbital skin ageing in participants of a melanoma case-control study in the U.K. Br J Dermatol. 2011, 165(5), 1011-21, DOI:10.1111/j.1365-2133.2011.10536.x.

11. Lupo, M.L.; Cohen, J.L.; Rendon, M.I. Novel Eye Cream Containing Mixture of Human Growth Factors and Cytokines for Treatment of Aged Skin around Eyes. J Am Acad Dermatol 2007, 56(2), 85, DOI:10.1016/j.jaad.2006.10.407.

12. Wagner, A.; Chaput, B.; Garrido, I.; Jalbert, F.; Grolleau, J.-L.; Chavoin, J.-P. Chirurgia estetica delle palpebre, EMC - Tecniche Chirurgiche - Chirurgia Plastica, Ricostruttiva ed Estetica 2013, 11(1), 1-10, DOI: 10.1016/S1769-6704(13)63949-2.

13. Coleman, S.R.; Grover, R. The Anatomy of the Aging Face: Volume Loss and Changes in 3-Dimensional Topography. Aesthetic Surg J 2006, 26(1), S4-S9, DOI:10.1016/j.asj.2005.09.012.

14. Gupta, M.A.; Gilchrest, B.A. Psychosocial Aspects Of Aging Skin. Dermatol Clinics 2005, 23(4), 643-648, DOI:10.1016/j.det.2005.05.012.

15. Sahni, K.; Kassir, M. Dermafrac ${ }^{\mathrm{TM}}$ : An Innovative New Treatment for Periorbital Melanosis in a Dark-Skinned Male Patient. J Cutan Aesthet Surg 2013, 6(3), 158-160, DOI:10.4103/0974-2077.118420.

16. Momosawa, A.; Kurita, M.; Ozaki, M.; Miyamoto, S.; Kobayashi, Y.; Ban, I.; Harii, K. Combined Therapy Using Q-Switched Ruby Laser and Bleaching Treatment with Tretinoin and Hydroquinone for Periorbital Skin Hyperpigmentation in Asians. Plastic \& Reconstructive Surg 2008, 121(1), 282 - 288, DOI:10.1097/01.prs.0000293869.00522.ec.

17. Verschoore, M.; Gupta, S.; Sharma, V.K.; Ortonne, J.P. Determination of Melanin and Haemoglobin in the Skin of Idiopathic Cutaneous Hyperchromia of the Orbital region (ICHOR): A Study of Indian Patients. J Cutan Aesthet Surg 2012, 5(3), 176-182, DOI:10.4103/0974-2077.101371.

18. Armanios, M.; Alder, J.K.; Parry, E.M.; Karim, B.; Strong, M.A.; Greider, C.W. Short Telomeres are Sufficient to Cause the Degenerative Defects Associated with Aging. Am J Human Gen 2009, 85(11), 823-832, DOI:10.1016/j.ajhg.2009.10.028.

19. Correia-Melo, C.; Hewitt, G.; Passos, J.F. Telomeres, oxidative stress and inflammatory factors: partners in cellular senescence? Longevity \& Healthspan 2014, 3(1), 1, DOI: 10.1186/2046-2395-3-1.

20. Ramos-e-Silva, M.; Carneiro, S. Elderly skin and its rejuvenation: Products and procedures for the aging skin, J Cosm Dermatol 2007, 6(1), 40-50, DOI:10.1111/j.1473-2165.2007.00289.x.

21. Kosmadaki, M.G.; Gilchrest, B.A. The role of telomeres in skin aging/photoaging. Micron 2004 35(3), 155-159, DOI:10.1016/j.micron.2003.11.002.

22. Suji, G.; Sivakami, S. Glucose, glycation and aging Biogerontology 2004, 5(6), 365-373, DOI:10.1007/s10522-004-3189-0.

23. Sude, K.M.; Venzke, K.; Mielke, H.; Breitenbach, U.; Mundt, C.; Jaspers, S.; Koop, U.; Sauermann, K.; Knußmann-Hartig, E.; Moll, I.; Gercken, G.; Young, A.R.; Stab, F.; Wenck, H.; Gallinat, S. Novel Aspects of Intrinsic and Extrinsic Aging of Human Skin: Beneficial Effects of Soy Extract, Photochemistry and Photobiology 2005, 81(3), 581-587, DOI:10.1562/2004-06-16-RA-202.1. 
24. Luebberding, S.; Krueger, N.; Kerscher, M. Mechanical properties of human skin in vivo: a comparative evaluation in 300 men and women. Skin Research and Technology 2014, 20(2), 127-135, DOI:10.1111/srt.12094

25. Farage, M.A.; Miller, K.W.; Berardesca, E.; Maibach, H.I. Clinical Implications of Aging Skin, Cutaneous Disorders in the Elderly. Am J Clin Dermatol 2009, 10(2), 73 - 86, DOI: 10.2165/00128071-200910020-00001.

26. Trojahn, C.; Dobos, G.; Lichterfeld, A.; Blume-Peytavi, U.; Kottner, J. Characterizing Facial Skin Ageing in Humans: Disentangling Extrinsic from Intrinsic Biological Phenomena. BioMed Res Int 2015, 2015, 1 - 9, DOI:10.1155/2015/318586.

27. Tzaphlidou, M. The role of collagen and elastin in aged skin: an image processing approach. Micron 2004, 35(3), 173 - 177, DOI:10.1016/j.micron.2003.11.003.

28. Hanson, M.D.; Chen, E. Daily Stress, Cortisol, and Sleep: The Moderating Role of Childhood Psychosocial Environments. Health Psychology 2010, 29(4), 394-402, DOI: 10.1037/a0019879.

29. Kimlin, M.G.; Guo, Y. Assessing the impacts of lifetime sun exposure on skin damage and skin aging using a non-invasive method. Sci Tot Env 2012, 425, 35-41, DOI:10.1016/j.scitotenv.2012.02.080.

30. Sugiyama-Nakagiri, Y.; Sugata, K.; Hachiya, A.; Osanai, O.; Ohuchi, A.; Kitahara, T. Ethnic differences in the structural properties of facial skin. J Derm Sci 2009, 53(2), 135-139, DOI:10.1016/j.jdermsci.2008.08.008.

31. Higuchi, T.; Satoh, T.; Yokozek, H.; Katayama, I.; Nishiok, K. Palpebral edema as a cutaneous manifestation of hyperthyroidism. J Am Acad Dermatology 2003, 48(4), 617-619, DOI:10.1067/mjd.2003.36.

32. Pelikan, Z. The possible involvement of nasal allergy in allergic keratoconjunctivitis. Eye 2009, 23, 1653-1660, DOI:10.1038/eye.2008.341

33. Pelikan, Z. Seasonal and perennial allergic conjunctivitis: the possible role of nasal allergy. Clinical $\mathcal{E}$ Experimental Ophthalmology 2009, 37, 448-457, DOI:10.1111/j.1442-9071.2009.02079.x.

34. Nettis, E.; Marcandrea, M.; Ferrannini, A.; Tursi, A. Tolerability of Nimesulide and Paracetamol in patients with NSAID- induced urticaria/angioedema. Immunopharmacology and Immunotoxicology 2001, 23(3), 343-354, DOI: 10.1081/IPH-100107335.

35. Mohaupt, M.G.; Vogt, B.; Frey, F.J. Sirolimus-associated eyelid edema in kidney transplant recipients. Transplantation 2001, 72(1), 162 - 164, DOI: 10.1097/00007890-200107150-00031.

36. Severino, G.; Chillotti, C.; De Lisa, R.; Del Zompo, M.; Ardau, R. Adverse Reactions During Imatinib and Lansoprazole treatment in Gastrointestinal Stromal Tumors. Ann Pharmacother 2005, 39(1), 162-164, DOI:10.1345/aph.1E127.

37. Maalouf, T.; Angiö̈, K.; Champigneulle, J.; Guerci, A.; George, J.L. Palpebral edema secondary to treatment by a specific inhibitor of tyrosine kinase: Glivec ${ }^{\circledR}$. A case report. J Fr Ophtalmole 2004, 27(1), 107-109, DOI:10.1016/S0181-5512(04)96103-7.

38. Zink, M.; Kuwilsky, A.; Knopf, U. Olanzapine-Associated Bilateral Eyelid Edema. J Clin Psychopharmacol 2007, 27(2), 214 - 215, DOI:10.1097/01.jcp.0000264968.69958.28.

39. Parsa, A.A.; Lye, K.D.; Radcliffe, N.; Parsa, F.D. Lower Blepharoplasty with Capsulopalpebral Fascia Hernia Repair for Palpebral Bags: A Long-Term Prospective Study, Plastic \& Reconstructive Surg 2008, 121(4), 1387-1397, DOI:10.1097/01.prs.0000304469.81239.f0.

40. Süer, K.H.; Kaptanoğlu, A.F. Association of Periorbital Edema and Fever in Acute Infectious Mononucleosis: A Case Report, Kafkas J Med Sci 2013, 3(3), 152-154, DOI:10.5505/kjms.2013.02886. 
41. Polder, K.D.; Landau, J.M.; Vergilis-Kalner, I.J.; Goldberg, L.H.; Friedman, P.M.; Bruce, S. Laser eradication of pigmented lesions: a review. Dermatol Surg 2011, 37(5), 572, DOI:10.1111/j.1524-4725.2011.01971.x.

42. Ma, G.; Lin, X.X.; Hu, X.J.; Jin, Y.B.; Chen, H. Treatment of venous infraorbital dark circles using a long-pulsed 1,064-nm neodymium-doped yttrium aluminum garnet laser. Dermatol Surg 2012, 38(8), 1277-82, DOI:10.1111/j.1524-4725.2012.02457.x.

43. Vavouli, C.; Katsambas, A.; Gregoriou, S.; Teodor, A.; Salavastru, C.; Alexandru, A.; Kontochristopoulos, G. Chemical peeling with trichloroacetic acid and lactic acid for infraorbital dark circles. J Cosmet Dermatol 2013, 12(3), 204-9, DOI:10.1111/jocd.12044.

44. Youn, S.; Shin, J.I.; Kim, J.D.; Kim, J.T.; Kim, Y.H. Correction of infraorbital dark circles using collagenase-digested fat cell grafts. Dermatol Surg 2013, 39(5), 766-772, DOI:10.1111/dsu.12140.

45. Friedmann, D.P.; Goldman, M.P. Dark Circles Etiology and Management Clin. Plastic Surg 2015, 42, 33 - 50, DOI:10.1016/j.cps.2014.08.007.

46. Vanaman, W.M.J.; Jones, I.T.; Bolton, J.; Larsen, L.; Wu, D.C.; Goldman, M.P. A Randomized, Investigator-Blinded Comparison of Two Topical Regimens in Fitzpatrick Skin Types III-VI With Moderate to Severe Facial Hyperpigmentation. J Drugs Dermatol 2017, 16(11), 1127-1132.

47. Poli, F. Hyperpigmentations du visage sur peaux mates et foncées, Cosmétologie, chirurgie dermatologique et spécificités dermatologiques sur peau noire. Ann Dermatol Vénéréol 2016, 143 (4), 28, DOI:10.1016/S0151-9638(16)30150-8.

48. Andrei, F.; Ersilia, A.; Tulcan, C.; Dragomirescu, A. Chemical Composition and the Potential of Lavandula angustifolia L. Oil as a Skin Depigmentant. Rec Nat Prod 2018, 12(4), 340-349, DOI:10.25135/rnp.36.17.10.061. 